\title{
Enhanced recombinant protein production and differential expression of molecular chaperones in sf-caspase-1-repressed stable cells after baculovirus infection
}

\author{
Yiu-Kay Lai ${ }^{1}$, John T-A Hsu ${ }^{2}$, Chih-Chieh Chu ${ }^{2}$, Teng-Yuan Chang ${ }^{2}$, Kao-Lu Pan ${ }^{2}$ and Chih-Chien Lin ${ }^{3 *}$
}

\begin{abstract}
Background: There are few studies that have examined the potential of RNA inference (RNAi) to increase protein production in the baculovirus expression vector system (BEVS). Spodoptera frugiperda (fall armyworm) (Sf)-caspase-1repressed stable cells exhibit resistance to apoptosis and enhancement of recombinant protein production. However, the mechanism of recombinant protein augmentation in baculovirus-infected Caspase-repressed insect cells has not been elucidated.

Results: In the current study, we utilized RNAi-mediated Sf-caspase-1-repressed stable cells to clarify how the resistance to apoptosis can enhance both intracellular (firefly luciferase) and extracellular (secreted alkaline phosphatase [SEAP]) recombinant protein production in BEVS. Since the expression of molecular chaperones is strongly associated with the maximal production of exogenous proteins in BEVS, the differential expression of molecular chaperones in baculovirus-infected stable cells was also analyzed in this study.

Conclusion: The data indicated that the retention of expression of molecular chaperones in baculovirus-infected Sfcaspase-1-repressed stable cells give the higher recombinant protein accumulation.
\end{abstract}

Keywords: Apoptosis, Baculovirus, Chaperone, RNA interference, Sf-caspase-1

\section{Background}

Programmed cell death, also known as apoptosis, is a normal physiological cell suicide program that is highly conserved among vertebrates and invertebrates [1,2]. Apoptotic cells undergo a series of dramatic and characteristic alterations in cellular morphology, such as DNA fragmentation, chromatin condensation, cytoskeleton reorganization, and plasma membrane blebbing [3]. Apoptosis plays an important role during development and tissue homeostasis eliminating discarded cells from the organism, including damaged and virus-infected cells. For this reason, apoptosis acts as a host protection mechanism by which virus-infected cells are removed to limit the proliferation of viruses $[4,5]$. Thus, to overcome this defense

\footnotetext{
* Correspondence: chchlin@pu.edu.tw

${ }^{3}$ Department of Cosmetic Science, Providence University, 200 Chung-Chi Road, Shalu, Taichung 43301, Taiwan, R.O.C

Full list of author information is available at the end of the article
}

response in cells, baculoviruses carry anti-apoptotic genes to inhibit programmed cell death [6-8].

Spodoptera frugiperda caspase-1 (Sf-caspase-1), the most studied effector caspase of Lepidoptera, is the principal effector caspase of S. frugiperda 9 (Sf9) cells, and is activated by various death stimuli, including baculovirus infection, ultraviolet (UV) irradiation, and over-expression of proapoptotic genes (Figure 1) [8-10]. Two types of antiapoptotic genes have been identified in baculoviruses, $p 35$ (p49) and inhibitor of apoptosis (IAP) [6-8]. The $p 35$ gene from Autographa californica multicapsid nucleopolyhedrovirus (AcMNPV) and $p 49$ gene from Spodoptera littoralis nucleopolyhedrovirus (SINPV) are required to prevent apoptosis during the virus infection of S. frugiperda cells, such as Sf9 and Sf21 $[2,11,12]$. The Op-IAP from the Orgyia pseudotsugata multicapsid nucleopolyhedrovirus (OpMNPV) and Sf-IAP from the host, S. frugiperda cells, also suppress the apoptosis process (Figure 1) $[1,2]$. 


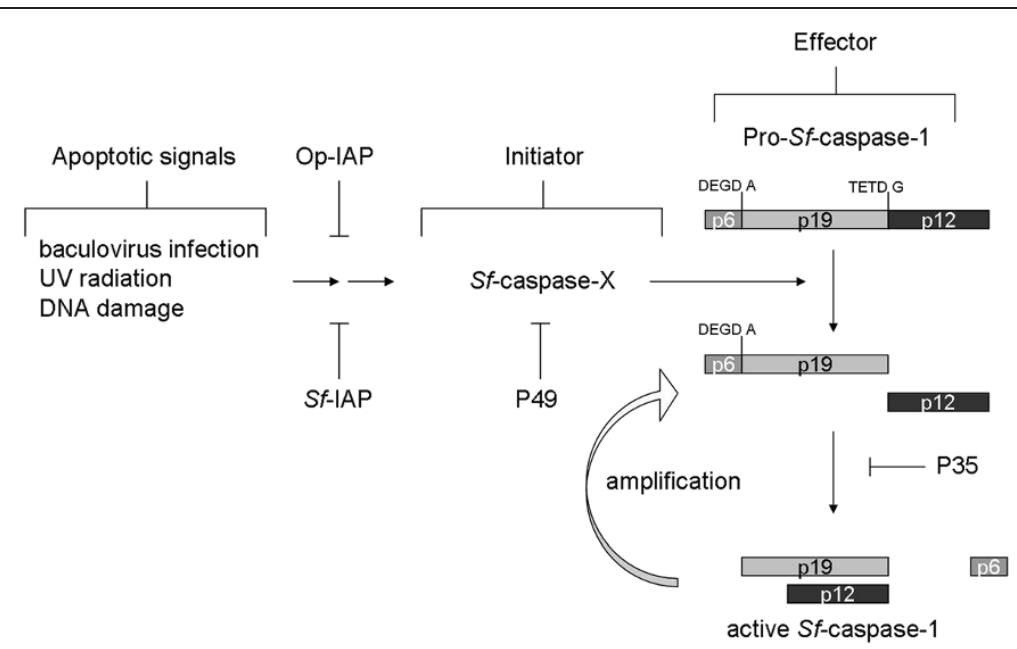

Figure 1 The apoptosis pathway in S. frugiperda cells. Apoptotic signals trigger the activation of initiator Sf-caspase-X in S. frugiperda cells. The Op-IAP and Sf-IAP suppress the activation of Sf-caspase-X, which proteolytically activates downstream effector caspases, including Sf-caspase1, by cleavage at the large-small subunit junction. The activated effector caspases can amplify this processing and different steps are inhibited by P49 and P35.

The baculovirus expression vector system (BEVS) is a powerful tool used for the expression of foreign proteins in numerous insect cells, including Sf9, Sf21 cells and Trichoplusi ni cells [13-15]. Therefore, recombinant protein production using a BEVS can be used for many pharmaceutical applications [16-18].

Exposure of cells to stressors, including high temperatures or a wide variety of physical and chemical insults, induces the expression of heat-shock proteins (HSPs) in cells $[19,20]$. HSPs are molecular chaperones responsible for maintaining cell homeostasis and promoting cell survival [21]. Baculovirus infection also serves as a stress factor that can activate both death-inducing and cellular-protective pathways, and the heat-shock response is important for baculovirus replication in insect cells [22]. Moreover, the rate-limited expression of endoplasmic reticular (ER) molecular chaperones is strongly associated with the maximal expression of exogenous proteins by BEVS [23].

Few studies have examined the potential of RNA inference (RNAi) to increase protein production in the BEVS $[24,25]$, however, several studies have demonstrated the efficiency of this approach in both insect cells and larvae $[26,27]$. In our previous studies, we used DNA vectorbased approaches with endogenously expressed doublestranded RNA (dsRNA) to silence its target gene, Sf-caspase-1, in Sf9 cells. Therefore, the Sf-caspase-1 mRNA level and plasmid copy number in the $S f$ caspase-1-repressed stable cells were examined [25]. In addition, $S f$-caspase-1-repressed stable cells exhibited resistance to apoptosis and enhancement of recombinant protein production $[25,28]$. These results were consistent with later findings in $T$. $n i$ cells [24]; however, the mechanism of recombinant protein augmentation in baculovirus-infected Caspase-repressed insect cells was not determined. Therefore, in this present study, we use RNAi-mediated Sf-caspase-1-repressed stable cells to clarify how resistance to apoptosis could enhance both intracellular (firefly luciferase) and extracellular (secreted alkaline phosphatase $[\mathrm{SEAP}]$ ) recombination protein production by BEVS. Furthermore, the differential expression of molecular chaperones in baculovirusinfected stable cells was also analyzed in this study.

\section{Results}

Stable transfection plasmid verification by genomic DNA PCR and RT-PCR in Sf9/plBdsCasp-1 and Sf9/plBdsCasp-2 cells

Stable cells (Sf9/pIB, Sf9/pIBdsCasp-1, and Sf9/pIBdsCasp-2 cells) were established by transfection of the control vector pIB or vector pIBdsCasp (Figure 2), and subsequently selected by BSD [25]. After more than 20 passages, stable cell lines were analyzed by genomic DNA PCR and RT-PCR to examine the inverted-repeat DNA sequence of $S f$-caspase-1 and endogenous expressed $S f$ caspase-1 mRNA quantity. The corresponding primers and PCR cycle number used for the experiments in Figure 3 are shown in Table 1. As shown for the genomic DNA PCR performed in Figure 3A, we supposed the pIBdsCasp vectors were stably integrated with Sf9 cellular genomic DNA. In addition, the data also showed that levels of Sf-casp1 mRNA in Sf9/pIBdsCasp-1 and Sf9/ pIBdsCasp-2 cells were apparently lower than those observed in Sf9 and Sf9/pIB cells (Figure 3B). Furthermore, control actin mRNA was not obviously affected in either of the cell lines. These results demonstrated that 

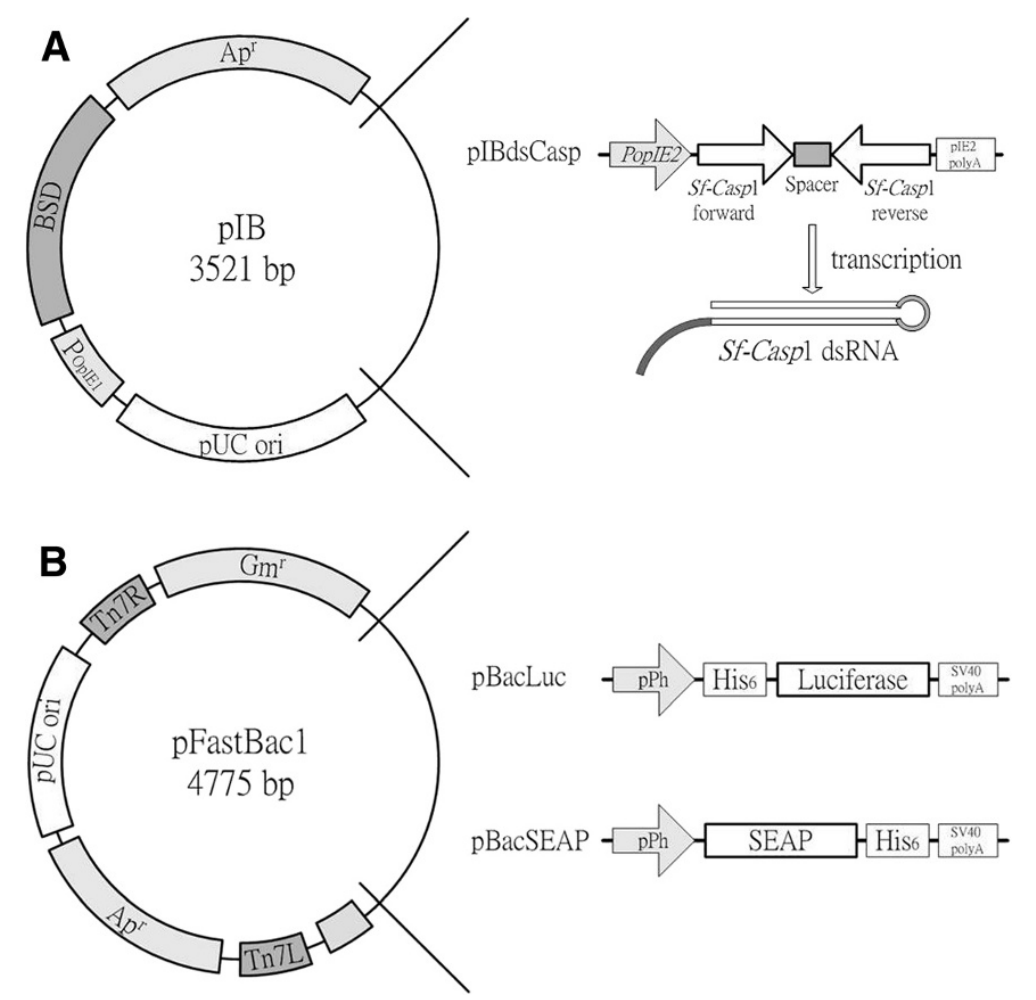

Figure 2 Inverted repeat vectors for the endogenous expression of Sf-casp1 dsRNA (A) and the recombinant baculovirus vectors (B). The resulting stably transfected cell lines carry a plasmid that contains an 800 bp stretch of DNA sequence of Sf-casp1 mRNA in both forward and reverse orientations, containing a spacer of 100 bp between each element. Recombinant baculovirus vectors, pBacLuc and pBacSEAP contained gene fragments encoding the luciferase and SEAP, respectively. Both genes were expressed under the control of the pPh promoter.

Sf-caspase-1 mRNA was successfully suppressed by $S f$ caspase-1 dsRNA in both Sf9/pIBdsCasp-1 and Sf9/ pIBdsCasp- 2 cells. In our previous study, we tested the expression of P35, a substrate inhibitor of Sf-caspase-1,

\begin{tabular}{|c|c|}
\hline \multicolumn{2}{|c|}{ A $\quad$ Genomic DNA PCR } \\
\hline & Sf9 plB dsCasp-1 dsCasp-2 \\
\hline \multicolumn{2}{|l|}{ plBdsCasp DNA } \\
\hline \multicolumn{2}{|l|}{ Sf- $\beta$-actin DNA } \\
\hline \multirow[t]{2}{*}{ B } & RT- PCR \\
\hline & plB dsCasp-1 dsCasp-2 \\
\hline Sf-casp1 mRNA & 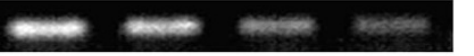 \\
\hline Sf- $\beta$-actin mRNA & 25 \\
\hline \multicolumn{2}{|c|}{$\begin{array}{l}\text { Figure } 3 \text { Genomic DNA PCR (A) and RT-PCR (B) analysis in Sf9/ } \\
\text { plBdsCasp-1 and Sf9/plBdsCasp-2 cells. Genomic DNA and total } \\
\text { RNA of the cells (Sf9, Sf9/plB, Sf9/plBdsCasp-1 and Sf9/plBdsCasp-2) } \\
\text { were extracted and analyzed by PCR and RT-PCR, respectively. The } \\
\text { corresponding specific primer sequences and PCR cycles are } \\
\text { presented in Table 1. plB, Sf9/plB cells; dsCasp-1, Sf9/plBdsCasp-1 } \\
\text { cells; dsCasp-2, Sf9/plBdsCasp-2 cells. }\end{array}$} \\
\hline
\end{tabular}

in Sf9/pIBdsCasp-1 and Sf9/pIBdsCasp-2 cells, and found that the level of P35 in pIBdsCasp transfected cells was evidently higher than that found in normal cells [25]. Thus, we suggested that $S f$-caspase-1 is suppressed in these $S f$-caspase-1-repressed cells.

\section{Accumulated SEAP expression by Sf9/pIBdsCasp-1 and Sf9/plBdsCasp-2 cells}

SEAP is a secreted (extracellular) recombinant protein that can be assayed in culture medium using a standard activity assay. Thus, we used rBacSEAP to infect the Sf9/ pIBdsCasp-1 and Sf9/pIBdsCasp-2 cells at a multiplicity of infection (MOI) of 0.1-100 and analyzed the accumulated SEAP expression in the culture medium every day post infection (dpi). At 2-4 dpi, the accumulated SEAP expression in rBacSEAP-infected Sf9/pIBdsCasp-1 and Sf9/pIBdsCasp- 2 cells was obviously higher than that in the control group, $\mathrm{Sf} 9$ and $\mathrm{Sf} 9 / \mathrm{pIB}$ cells, at all MOIs (Figure 4). Moreover, at an MOI of 1 and 10, accumulated SEAP expression in rBacSEAP-infected Sf9/pIBdsCasp-1 and Sf9/pIBdsCasp-2 cells was approximately two-fold higher than that in the control group after 4 dpi (Figure 4B and 4C). Moreover, the difference of accumulated SEAP expression between normal and 
Table 1 Primers used in PCR and RT-PCR analyses

\begin{tabular}{|c|c|c|c|c|c|c|}
\hline Gene & Protein/product & Predicted location & Description & Primer sequence & PCR cycle & Reference \\
\hline \multirow[t]{2}{*}{$\overline{\text { Sf-casp } 1}$} & Sf-casp1 dsRNA & nucleus/cytosol & plBdsCasp DNA, forward & 5'-GATATGGAGAAGATCTGGCAC-3' & 35 & {$[25]$} \\
\hline & & & plBdsCasp DNA, reverse & 5'-GTGGGGCAGGGCGTAGCC-3' & & \\
\hline \multirow[t]{2}{*}{ Sf- $\beta$-actin } & $\beta$-Actin & cytosol & Sf- $\beta$-actin DNA/mRNA, forward & 5'-CAATCTGTCACCTTGGCA-3' & 25 & [25] \\
\hline & & & Sf- $\beta$-actin DNA/mRNA, reverse & 5'-GACAATACAAACTAAGATTTA-3' & & \\
\hline \multirow[t]{2}{*}{ Sf-casp 1} & Sf-Caspase-1 & cytosol & Sf-casp 1 mRNA, forward & 5'-TGTCAAACACCTITTATG-3' & 25 & {$[25]$} \\
\hline & & & Sf-casp1 mRNA, reverse & 5'-TATTATGACACATGGGCA-3' & & \\
\hline \multirow[t]{2}{*}{$a+x 1$} & Copper chaperone & cytosol & atx1 mRNA, forward & $5^{\prime}$-ATGTCATCTACACACATTTT-3' & 25 & [29] \\
\hline & & & atx1 mRNA, reverse & 5'-CTATTGTGTGCCAACATAAG-3' & & \\
\hline \multirow[t]{2}{*}{ cypA } & Cyclophilin A (CypA) & cytosol & cypA mRNA, forward & 5'-ATGGCTITACCCCGAGTTT-3' & 25 & [29] \\
\hline & & & cypA mRNA, reverse & 5'-TGGTTTGTGAAGTCACCTCCT-3' & & \\
\hline \multirow[t]{2}{*}{ сур B } & Cyclophilin B (CypB) & secreted/ER & cypB mRNA, forward & 5'-GCGCTTTCGCAGCAGCTCTT-3' & 25 & [29] \\
\hline & & & cypB mRNA, reverse & 5'-AGCCCTGGCCTTCTGGTTT-3' & & \\
\hline \multirow[t]{2}{*}{ hsc70 } & Heat shock cognate & ER & hsc70 mRNA, forward & 5'-ATGATCAAAATGCGGTGGA-3' & 25 & {$[30]$} \\
\hline & 70 (GRP78-like) & & hsc70 mRNA, reverse & 5'-AGCCACATATGAGGGAGTGAT-3' & & \\
\hline \multirow[t]{2}{*}{ hsp90 } & Heat shock protein & ER & hsp90 mRNA, forward & 5'-ATGCCCGAAGAAATGCAG-3' & 25 & {$[31]$} \\
\hline & 90 (HSP90) & & hsp90 mRNA, reverse & 5'-TGTACAGCTCCTTGCCGCT-3' & & \\
\hline \multirow[t]{2}{*}{ calr } & Calreticulin (CRT) & cytosol & calr mRNA, forward & 5'-ATTCTATTCGTGGCCAGTCC-3' & 25 & [29] \\
\hline & & & calr mRNA, reverse & 5'-GAACTITCCAGCAGTCAGCTT-3' & & \\
\hline \multirow[t]{2}{*}{ pdi } & Protein disulfide & ER & pdi mRNA, forward & 5'-GCCATTGACGCTGACGAA-3' & 25 & {$[29]$} \\
\hline & isomerase (PDI) & & pdi mRNA, reverse & $5^{\prime}$-CCTCGCTCAACAAGTGCTGT-3' & & \\
\hline
\end{tabular}

$\mathrm{ER}$, endoplasmic reticulum.

Sf-caspase-1-repressed stable cells increased with MOI, such as that at MOIs of 10 and 100 (Figure 4). These data demonstrated that the secreted recombinant protein production in $S f$-caspase-1-repressed stable cells was higher than that in normal insect cell after baculovirus infection.

\section{Luciferase expression in Sf9/pIBdsCasp-1 and Sf9/ plBdsCasp-2 cells}

To estimate different kinds of recombinant protein production in Sf-caspase-1-repressed stable cells, we use an intracellular recombinant protein, luciferase, as the assay target. The rBacLuc-infected Sf9/pIBdsCasp-1 and Sf9/ pIBdsCasp-2 cells showed higher luciferase activities than that in control cells at all MOIs at 2 or 3 dpi (Figure 5). However, at all MOIs, the difference of luciferase expression was not apparent at $>5$ dpi (Figure 5) and might result from the lysis of virus-infected cells during the later phase of infection releasing intracellular proteins [32].

\section{SEAP expression at each day's period in Sf9/plBdsCasp-1 and Sf9/plBdsCasp-2 cells}

To further investigate the differences of recombinant protein production between the normal and $S f$-caspase1 -repressed stable cells, specific SEAP activity at every 24 hour period of time in the culture media of rBacSEAP infected cells was analyzed. At an MOI of 0.1 and
1, SEAP activities in culture medium of Sf9/pIBdsCasp-1 and Sf9/pIBdsCasp- 2 cells were higher than that of normal cells at 3-5 dpi (Figure 6A and 6B). Moreover, at an MOI of 10 and 100, peak SEAP expression in Sfcaspase-1-repressed and normal cells occurred at 2-4 dpi (Figure 6C and 6D). Although during the assay the replacement of culture medium of virus-infected cells may affect not only the energy sources for protein expression but also virus replication in cells, the difference of recombinant protein production between Sf-caspase-1repressed and normal cells may be caused by the diverse cellular states that occur during the middle phase of the infection process.

\section{Expression of molecular chaperones in baculovirus- infected Sf-caspase-1-repressed stable cells}

The heat shock response is important for baculovirus replication in insect cells and the expression of ER molecular chaperones is strongly associated with the maximal expression of exogenous proteins in BEVS [22,23]. Therefore, we analyzed the mRNA expression of some molecular chaperones involved in virus replication, including copper chaperone, cyclophilins, HSPs, calreticulin (CRT), and protein disulfide isomerase (PDI). The $\beta$-actin mRNA level in Sf9 and Sf9/pIBdsCasp-1 cells was relatively equivalent at each time point analyzed and decreased at 9-48 hours post-infection (hpi) (Figure 7B). However, the mRNA 


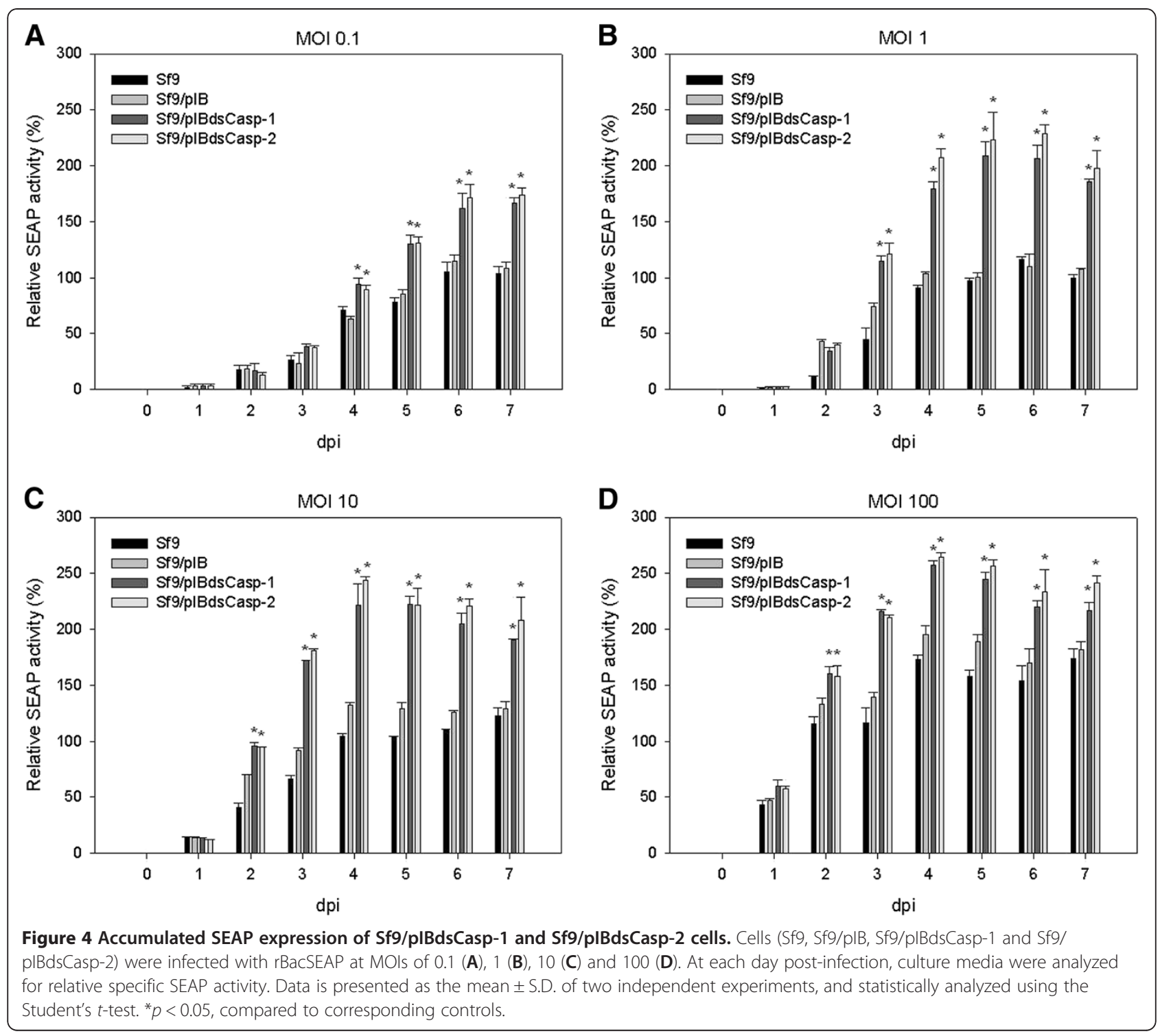

expression of chaperones, including atx1, cyp $A, \operatorname{cyp} B$, $h s c 70$, calr, and pdi, exhibited differential expression between baculovirus-infected Sf9 and Sf9/pIBdsCasp-1 cells at 9-48 hpi (Figure 7). Most chaperones demonstrated an induction response for virus infection within 9 hpi. Furthermore, expression of $h s p 90$ was persistent during the infection process and no dramatic difference was observed between normal and $S f$-caspase-1-repressed cells (Figure 7G). In contrast, expression of $h s c 70$ did not show the same stable expression (Figure 7E). These data are consistent with a study performed by Nobiron et al. [33] that identified the cause of this effect to be HSC70, a virus-induced member of the HSP70 family.

\section{Discussion}

In this study, our data demonstrated that both intracellular (luciferase) and extracellular (SEAP) recombinant protein production in $S f$-caspase-1-repressed stable cells was higher than that in normal insect cell after baculovirus infection (Figure 4 and 5). Although luciferase expression did not correlate with accumulated SEAP expression, it was apparent that both extracellular and intracellular recombinant protein production in $S$ caspase-1-repressed stable cells was higher than for parental cells (Figures 4 and 5). Thus, these data indicate that $S f$-caspase-1-repressed stable cells express a higher amount of recombinant protein in BEVS, consistent with previous studies in S. frugiperda cells by our group [25] and in T. ni cells by Bentley's group [24,34]. Hence, we can suggest that the apoptotic repressed insect cells have greater recombinant protein production when infected with recombinant baculovirus, providing an effective production tool for BEVS. Besides, results also indicated that the difference of recombinant protein 


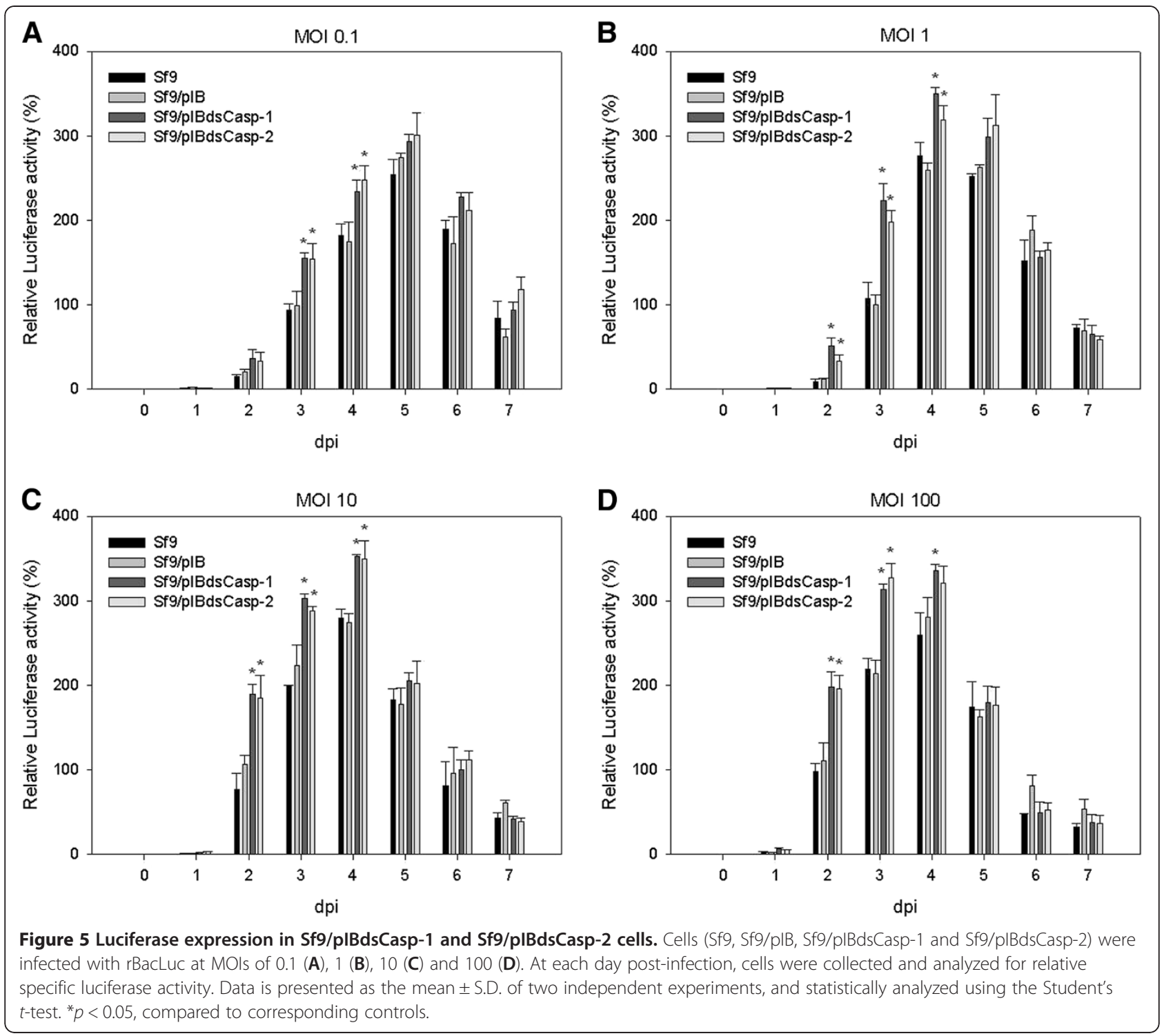

production between $S f$-caspase-1-repressed and normal cells may be caused by the diverse cellular states that occur during the middle phase of the infection process (Figure 6).

Molecular chaperones are important and highly associated with the state of cells. The results of molecular chaperone mRNA levels in baculovirus-infected normal and $S f$-caspase-1-repressed stable cells demonstrated that these cells were appeared in different states during the infection progression (Figure 7). Therefore, we assumed that this is a key explanation for baculovirusinfected $S$-caspase-1-repressed stable cells have a higher recombinant protein production than that in normal cells. The persistent expression of molecular chaperones in baculovirus-infected $S f$-caspase-1-repressed stable cells resulted in a higher recombinant protein production than that in normal cells, which can be suggested by some earlier studies that focused on the co-expression of molecular chaperones, Bip (GRP78), Calreticulin and Calnexin in BEVS to improve the recombinant production and secretion from cells and larvas [35-38]. Therefore, we also suppose that expression of molecular chaperon in combined with RNAi technique may have opportunity to further improve BEVS.

During the baculovirus infection process, the condition of infected insect cells often represents key factors that affect both baculovirus replication and recombinant production. Therefore, there appears to be a distinct difference of the baculovirus infection process that occurs in normal and $S$-caspase-1-repressed cells. We proposed that the $S f$-caspase-1-repressed stable cells have a different status and this response improves the ability of infected cells to express a higher amount of recombinant proteins. 


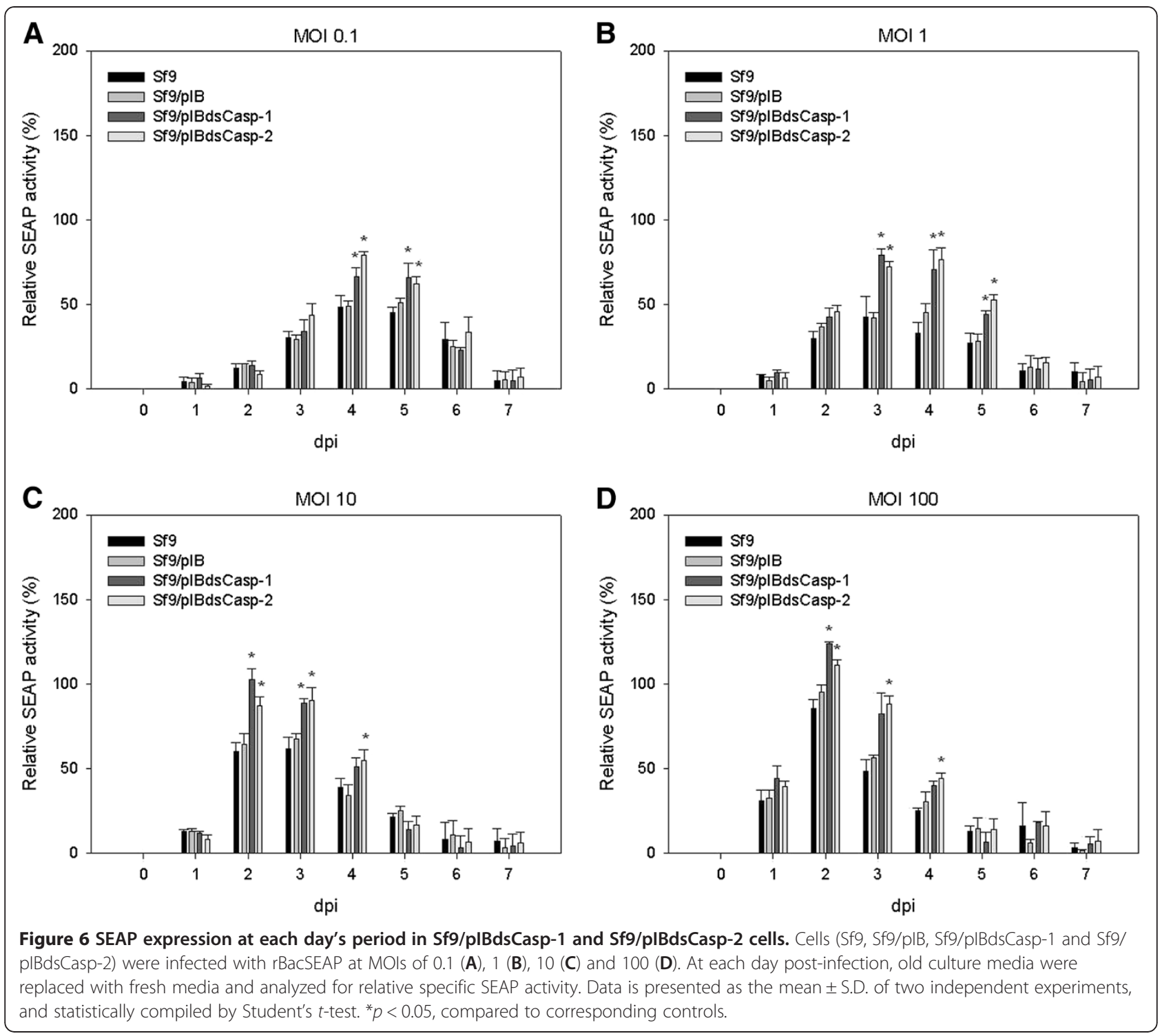

\section{Conclusions}

In summary, the differential expression of molecular chaperones in baculovirus-infected $S f$-caspase-1-repressed stable cells affects the production of recombinant protein when compare with normal cells. Therefore, the current study identified critical virus-cell interactions that are likely to improve the development of BEVS in future studies.

\section{Methods}

Construction of plasmids and recombinant baculovirus

Stable transfection plasmids were constructed using the pIB vector (Invitrogen, Carlsbad, CA) as the backbone. The 5 ' end 800 base pairs (bp) forward and reverse DNA fragments of the $S f$-caspase-1 ( $S f$-casp 1$)$ gene were amplified by polymerase chain reaction (PCR) from the genomic DNA extracted from Sf9 cells. The resulting construct was named pIBdsCasp, containing an inverted-repeat sequence of $S f$-casp 1 under the control of the opIE2 promoter, as described previously [25] and shown in Figure 2A. Illustrations of pBacLuc and pBacSEAP are provided in Figure 2B, and the construction of these plasmids was performed using the Bac-to-Bac system according to the manufacturer's protocols (Invitrogen) as described previously $[25,28]$. Luciferase and SEAP were expressed in rBacLuc-, and rBacSEAPinfected insect cells, respectively. Recombinant baculovirus were amplified twice and titers were determined by the end-point dilution method [39].

\section{Cell culture and transfection}

Sf9 cells were cultured in Grace's insect cell culture medium (Invitrogen) with 10\% (v/v) heat inactivated fetal bovine serum (FBS; Hyclone, Logan, UT) at $27^{\circ} \mathrm{C}$. 

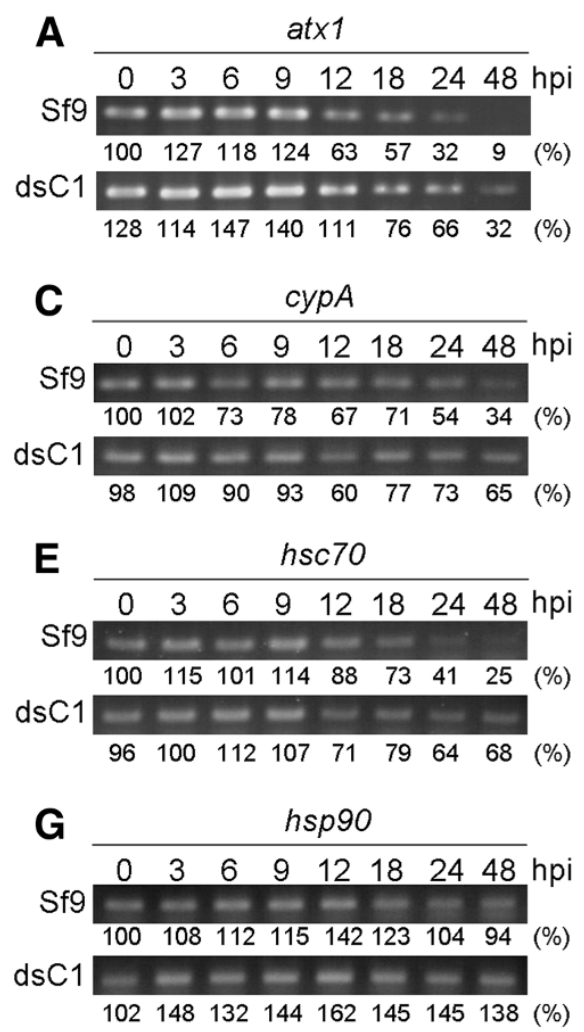
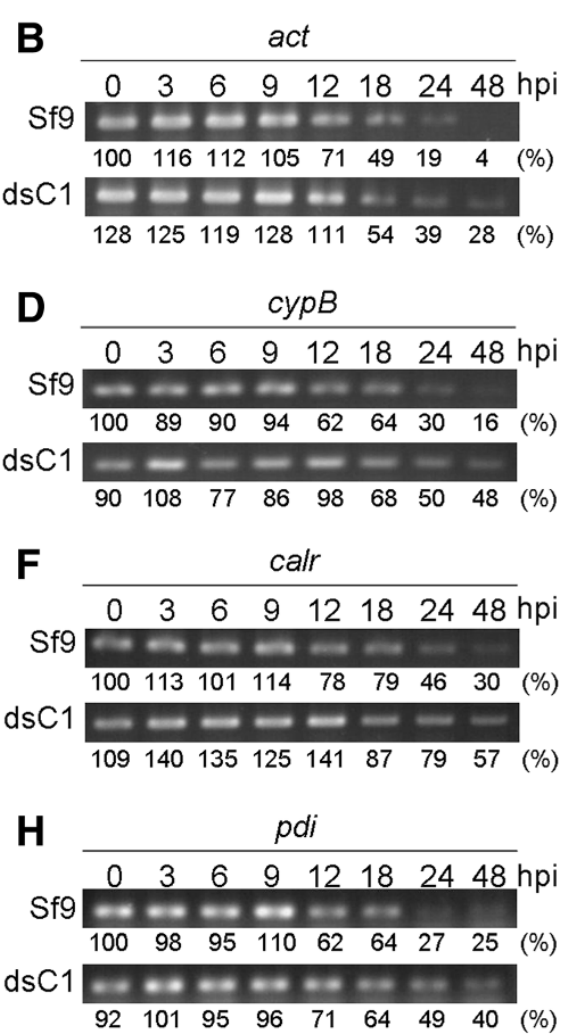

Figure 7 RT-PCR analysis of molecular chaperones in baculovirus-infected Sf-caspase-1-repressed stable cells. Total mRNA of baculovirusinfected cells, Sf9 and Sf9/plBdsCasp-1, at each hour post infection (hpi) were extracted and analyzed by RT-PCR. The marked numbers below images were relative mRNA level (\%), which were normalized with rRNA and compared with corresponding mRNA level of Sf9 cell at 0 hpi. The corresponding specific primer sequences and PCR cycles were presented in Table 1. dsC1, Sf9/plBdsCasp-1 cells; act, $\beta$-actin.

Cell density was determined by hemocytometer counts and cell viability was evaluated by the Trypan Blue exclusion method. The pIB vector or pIBdsCasp plasmids were transfected into Sf9 cells by Transfast reagent (Promega, Madison, WI) and stable cell lines were selected by the addition of $60 \mu \mathrm{g}$ Blasticidin (BSD)/mL (Invitrogen), as previously described [25]. Stable clones encoding $\mathrm{pIB}$ vector and $\mathrm{pIBdsCasp}$ plasmid were designated Sf9/pIB and Sf9/pIBdsCasp (Sf9/pIBdsCasp-1 and Sf9/ pIBdsCasp-2) cells, respectively.

\section{PCR and RT-PCR analysis}

Primers used for PCR and reverse transcription PCR (RT-PCR) analyses are listed in Table 1. The genomic DNA was extracted from $5 \times 10^{4}$ stable insect cells using the Dneasy Tissue Extraction kit (Qiagen, Hilden, Germany) and 500 ng of extracted genomic DNA was used in each $50 \mu \mathrm{L} \mathrm{PCR}$. For genomic DNA PCR analysis, after an initial incubation at $94^{\circ} \mathrm{C}$ for $4 \mathrm{~min}$, the reaction mixture was subjected to 25 or 35 cycles (Table 1) of PCR amplification at $94^{\circ} \mathrm{C}$ for $15 \mathrm{~s}$, at $55^{\circ} \mathrm{C}$ for $30 \mathrm{~s}$, and at $72^{\circ} \mathrm{C}$ for $1 \mathrm{~min}$. PCR products were resolved by $1 \%$ agarose gel electrophoresis and analyzed after ethidium bromide staining.
Total RNA were isolated from cells using Trizol reagent (Invitrogen) according to standard protocols [40] and RNA concentrations were determined by UV absorption at $260 \mathrm{~nm}$. To avoid DNA contamination, RNA samples were treated with RNase-free DNase I (Invitrogen) for $20 \mathrm{~min}$ at $37^{\circ} \mathrm{C}$. For each RT-PCR, $200 \mathrm{ng}$ of RNA were used in a $50 \mu \mathrm{L}$ reaction. A reverse transcription step $\left(50^{\circ} \mathrm{C}\right.$ for $30 \mathrm{~min}$ and $94^{\circ} \mathrm{C}$ for $\left.2 \mathrm{~min}\right)$ was followed by 25 cycles (Table 1 ) of denaturation at $94^{\circ} \mathrm{C}$ for $15 \mathrm{~s}$, annealing at $55^{\circ} \mathrm{C}$ for $30 \mathrm{~s}$, and extension at $72^{\circ} \mathrm{C}$ for $1 \mathrm{~min}$. RT-PCR products were resolved by $1.2 \%$ agarose gel electrophoresis and analyzed after ethidium bromide staining. The ribosomal RNA (rRNA) of insect cells served as a loading control in the RT-PCR analysis.

\section{Luciferase and SEAP activity analyses}

For luciferase activity analysis, enzyme activity in the cell lysate from $2 \times 10^{4}$ cells was determined by the Luciferase reporter assay system (Promega) according to the manufacturer's procedures and analyzed by a Victor ${ }^{2}$ Multilabel Counter (PerkinElmer Life Sciences, Norwalk, CT). Specific luciferase activities are presented as mean values normalized against those of the control (infected Sf9 cells). 
For SEAP activity analysis, production of SEAP in $10 \mu \mathrm{L}$ cultured medium was quantified by using a pnitrophenylphosphate-based (pNPP) light absorbance time course method [41] and also analyzed by a Victor ${ }^{2}$ Multilabel Counter. The specific SEAP activities are presented as mean percentage relative to control (infected Sf9 cells).

\section{Statistical analysis}

Quantitative data were analyzed using Student's $t$-test and presented as means \pm standard deviation (S.D.) of two or three independent experiments. The $P$-values $<0.05$ were considered significant.

\section{Competing interests}

The authors declare that they have no competing interests.

\section{Authors' contributions}

YKL and JTAH designed the approach, helped with experimental design and data analysis, participated with manuscript preparation and editing. CCC, TYC and KLP helped with experiments execution, provided bioinformatics research and participated with manuscript preparation and editing. CCL participated with experiments execution and data interpretation, participated with prepared and submitted the manuscript. All authors read and approved the final manuscript.

\section{Acknowledgements}

This work was supported by grants from the National Science Council, Taiwan, R.O.C. (NSC99-2313-B-126-002-MY3).

\section{Author details}

${ }^{1}$ Institute of Biotechnology, Department of Life Science, National Tsing Hua University, No. 101, Section 2, Kuang-Fu Road, Hsinchu 30013, Taiwan, R.O.C. ${ }^{2}$ Division of Biotechnology and Pharmaceutical Research, National Health Research Institutes, 35 Keyan Road, Zhunan, Miaoli 35053, Taiwan, R.O.C. ${ }^{3}$ Department of Cosmetic Science, Providence University, 200 Chung-Chi Road, Shalu, Taichung 43301, Taiwan, R.O.C.

Received: 22 June 2012 Accepted: 4 November 2012

Published: 7 November 2012

\section{References}

1. Schafer ZT, Kornbluth S: The apoptosome: physiological, developmental, and pathological modes of regulation. Dev Cell 2006, 10:549-561.

2. Zoog SJ, Schiller JJ, Wetter JA, Chejanovsky N, Friesen PD: Baculovirus apoptotic suppressor P49 is a substrate inhibitor of initiator caspases resistant to P35 in vivo. EMBO J 2002, 21:5130-5140.

3. Ferri KF, Kroemer G: Organelle-specific initiation of cell death pathways. Nat Cell Biol 2001, 3:E255-E263.

4. Kim YS, Xiao HZ, Du EQ, Cai GS, Lu SY, Qi YP: Identification and functional analysis of LsMNPV anti-apoptosis genes. J Biochem Mol Biol 2007, 40:571-576.

5. Pei Z, Reske G, Huang Q, Hammock BD, Qi Y, Chejanovsky N: Characterization of the apoptosis suppressor protein P49 from the Spodoptera littoralis nucleopolyhedrovirus. J Biol Chem 2002, 277:48677-48684.

6. Manji GA, Friesen PD: Apoptosis in motion. An apical, P35-insensitive caspase mediates programmed cell death in insect cells. J Biol Chem 2001, 276:16704-16710.

7. LaCount DJ, Hanson SF, Schneider CL, Friesen PD: Caspase inhibitor P35 and inhibitor of apoptosis Op-IAP block in vivo proteolytic activation of an effector caspase at different steps. J Biol Chem 2000, 275:15657-15664.

8. Seshagiri S, Miller LK: Baculovirus inhibitors of apoptosis (IAPs) block activation of Sf-caspase-1. Proc Natl Acad Sci 1997, 94:13606-13611.

9. Bideshi DK, Tan Y, Bigot Y, Federici BA: A viral caspase contributes to modified apoptosis for virus transmission. Genes Dev 2005, 19:1416-1421.

10. Ahmad M, Srinivasula SM, Wang L, Litwack G, Fernandes-Alnemri T, Alnemri ES: Spodoptera frugiperda caspase-1, a novel insect death protease that cleaves the nuclear immunophilin FKBP46, is the target of the baculovirus antiapoptotic protein p35. J Biol Chem 1997, 272:1421-1424.
11. Yu M, Li Z, Yang K, Lin T, Gong Y, Pan L, Pang Y: Identification of the apoptosis inhibitor gene p49 of Spodoptera litura multicapsid nucleopolyhedrovirus. Virus Genes 2005, 31:145-151.

12. Means JC, Muro I, Clem RJ: Silencing of the baculovirus Op-iap3 gene by RNA interference reveals that it is required for prevention of apoptosis during Orgyia pseudotsugata $\mathrm{M}$ nucleopolyhedrovirus infection of Ld652Y cells. J Virol 2003, 77:4481-4488.

13. Zhu B, Ye J, Lu P, Jiang R, Yang X, Fu ZF, Chen H, Cao S: Induction of antigen-specific immune responses in mice by recombinant baculovirus expressing premembrane and envelope proteins of West Nile virus. Virol J 2012, 9:132.

14. Lynch AG, Tanzer F, Fraser MJ, Shephard EG, Williamson AL, Rybicki EP: Use of the piggyBac transposon to create HIV-1 gag transgenic insect cell lines for continuous VLP production. BMC Biotechnol 2010, 10:30.

15. Y-c H: Baculovirus as a highly efficient expression vector in insect and mammalian cells. Acta Pharmacol Sin 2005, 26:405-416.

16. Kost TA, Condreay JP, Jarvis DL: Baculovirus as versatile vectors for protein expression in insect and mammalian cells. Nat Biotechnol 2005, 23:567-575

17. Cox MM: Recombinant protein vaccines produced in insect cells. Vaccine 2012, 30:1759-1766.

18. van Oers MM: Opportunities and challenges for the baculovirus expression system. J Invertebr Pathol 2011, 107(Suppl):S3-S15.

19. Huang $\mathrm{KL}$, Lai YK, Lin CC, Chang JM: Involvement of GRP78 in inhibition of HBV secretion by Boehmeria nivea extract in human HepG2 2.2.15 cells. J Viral Hepat 2009, 16:367-375.

20. Chang YS, Lo CW, Sun FC, Chang MD, Lai YK: Differential expression of Hsp90 isoforms in geldanamycin-treated 9L cells. Biochem Biophys Res Commun 2006, 344:37-44.

21. Shu CW, Sun FC, Cho JH, Lin CC, Liu PF, Chen PY, Chang MD, Fu HW, Lai YK: GRP78 and Raf-1 cooperatively confer resistance to endoplasmic reticulum stress-induced apoptosis. J Cell Physiol 2008, 215:627-635.

22. Lyupina $\mathrm{V}$, Dmitrieva SB, Timokhova AV, Beljelarskaya SN, Zatsepina OG, Evgen'ev MB, Mikhailov VS: An important role of the heat shock response in infected cells for replication of baculoviruses. Virology 2010, 406:336-341.

23. Yun EY, Goo TW, Kim SW, Choi KH, Hwang JS, Kang SW, Kwon OY: Changes in cellular secretory processing during baculovirus infection. Biotechnol Lett 2005, 27:1041-1045.

24. Hebert CG, Valdes JJ, Bentley WE: In vitro and in vivo RNA interference mediated suppression of Tn-caspase-1 for improved recombinant protein production in High Five cell culture with the baculovirus expression vector system. Biotechnol Bioeng 2009, 104:390-399.

25. Lin CC, Hsu JT, Huang KL, Tang HK, Shu CW, Lai YK: Sf-Caspase-1-repressed stable cells: resistance to apoptosis and augmentation of recombinant protein production. Biotechnol Appl Biochem 2007, 48:11-19.

26. Kim EJ, Kramer SF, Hebert CG, Valdes JJ, Bentley WE: Metabolic engineering of the baculovirus-expression system via inverse "shotgun" genomic analysis and RNA interference (dsRNA) increases product yield and cell longevity. Biotechnol Bioeng 2007, 98:645-654.

27. Kramer SF, Bentley WE: RNA interference as a metabolic engineering tool: potential for in vivo control of protein expression in an insect larval model. Metab Eng 2003, 5:183-190.

28. Lin CC, Hsu JT, Huang KL, Tang HK, Lai YK: Stable RNA interference in Spodoptera frugiperda cells by a DNA vector-based method. Biotechnol Lett 2006, 28:271-277.

29. Negre V, Hotelier T, Volkoff AN, Gimenez S, Cousserans F, Mita K, Sabau $X$, Rocher J, Lopez-Ferber M, D'Alencon E, et al: SPODOBASE: an EST database for the lepidopteran crop pest Spodoptera. BMC Bioinforma 2006, 7:322.

30. Popham HJ, Grasela JJ, Goodman CL, Mclntosh AH: Baculovirus infection influences host protein expression in two established insect cell lines. J Insect Physiol 2010, 56:1237-1245.

31. Landais I, Pommet J, Mita K, Nohata J, Gimenez S, Fournier P, Devauchelle $\mathrm{G}$, Duonor-Cerutti $M$, Ogliastro M: Characterization of the cDNA encoding the $90 \mathrm{kDa}$ heat-shock protein in the Lepidoptera Bombyx mori and Spodoptera frugiperda. Gene 2001, 271:223-231.

32. Wu SC, Huang H, Lin CC: Expression and functional characterization of Helicobacter pylori catalase from baculovirus-infected insect cells. Enzyme Microb Technol 2004, 35:482-487.

33. Nobiron I, O'Reilly DR, Olszewski JA: Autographa californica nucleopolyhedrovirus infection of Spodoptera frugiperda cells: a global 
analysis of host gene regulation during infection, using a differential display approach. J Gen Virol 2003, 84:3029-3039.

34. Hebert CG, Valdes JJ, Bentley WE: Investigating apoptosis: characterization and analysis of Trichoplusia ni-caspase-1 through overexpression and RNAi mediated silencing. Insect Biochem Mol Biol 2009, 39:113-124.

35. Zhang L, Wu G, Tate CG, Lookene A, Olivecrona G: Calreticulin promotes folding/dimerization of human lipoprotein lipase expressed in insect cells (sf21). J Biol Chem 2003, 278:29344-29351.

36. Hsu TA, Betenbaugh MJ: Coexpression of molecular chaperone BiP improves immunoglobulin solubility and IgG secretion from Trichoplusia ni insect cells. Biotechnol Prog 1997, 13:96-104.

37. Sokolenko S, George S, Wagner A, Tuladhar A, Andrich JM, Aucoin MG Co-expression vs. co-infection using baculovirus expression vectors in insect cell culture: Benefits and drawbacks. Biotechnol Adv 2012, 30:766-781.

38. Nakajima M, Kato T, Kanamasa S, Park EY: Molecular chaperone-assisted production of human alpha-1,4-N-acetylglucosaminyltransferase in silkworm larvae using recombinant BmNPV bacmids. Mol Biotechnol 2009, 43:67-75.

39. O'Reilly D, Miller L, Luckow V: Baculovirus expression vectors: A laoratory manual. NY: WH Freeman and Co.; 1992.

40. Huang KL, Lai YK, Lin CC, Chang JM: Inhibition of hepatitis B virus production by Boehmeria nivea root extract in HepG2 2.2.15 cells. World J Gastroenterol 2006, 12:5721-5725.

41. Berger J, Hauber J, Hauber R, Geiger R, Cullen BR: Secreted placental alkaline phosphatase: a powerful new quantitative indicator of gene expression in eukaryotic cells. Gene 1988, 66:1-10.

doi:10.1186/1472-6750-12-83

Cite this article as: Lai et al:: Enhanced recombinant protein production and differential expression of molecular chaperones in sf-caspase-1repressed stable cells after baculovirus infection. BMC Biotechnology 2012 12:83.

\section{Submit your next manuscript to BioMed Central and take full advantage of:}

- Convenient online submission

- Thorough peer review

- No space constraints or color figure charges

- Immediate publication on acceptance

- Inclusion in PubMed, CAS, Scopus and Google Scholar

- Research which is freely available for redistribution 